\title{
AD-0377 - Role of educational level on the prevalence of diabetes, obesity and hypertension among Bulgarian Roma people
}

1Stefan Krivoshiev, 2Anna-Maria Borissova, 3Alexander Shinkov, 1Anna Koteva, 1Georgy Mihaylov, 1Zdravko Kraev, 4Petar Shikov, 2Todor Todorov. 1University Hospital Queen Jovanna, Medical University of Sofia, Sofia, Bulgaria; 2University Hospital Sofiamed, Sofia University "Kliment Ohridsky", Bulgaria; 3University Hospital of Endocrinology, Medical University-Sofia, Bulgaria; 4District Hospital Pazardjik, Bulgaria

Roma people comprise about $5 \%$ of the whole population in Bulgaria. They are less educated, most of them are unemployed, without health insurance. It is well known that the mean life span of Roma people is at least 5 years shorter compared to the remaining Bulgarian population. Chronic diseases among them are more prevalent but this observation has not been statistically verified. Until now there is a complete lack of confirmed data on the frequency of some chronic diseases among this minority.

The aim of the screening program was to investigate the prevalence of diabetes, hypertension and obesity in relation of educational level among Roma people in Bulgaria.

Material and methods: After receiving informed consent 365 Roma people (88 male, $24 \%$ and 277 female, $76 \%$ ), mean age $43.9 \pm 16.1$ y (18 to $88 \mathrm{y}$ ) were screened. We collected data on educational level, presence of arterial hypertension and diabetes. Actual arterial pressure was measured, BMI was calculated and fasting blood sugar was determined. The data were processed statistically using SPSS 13.0.

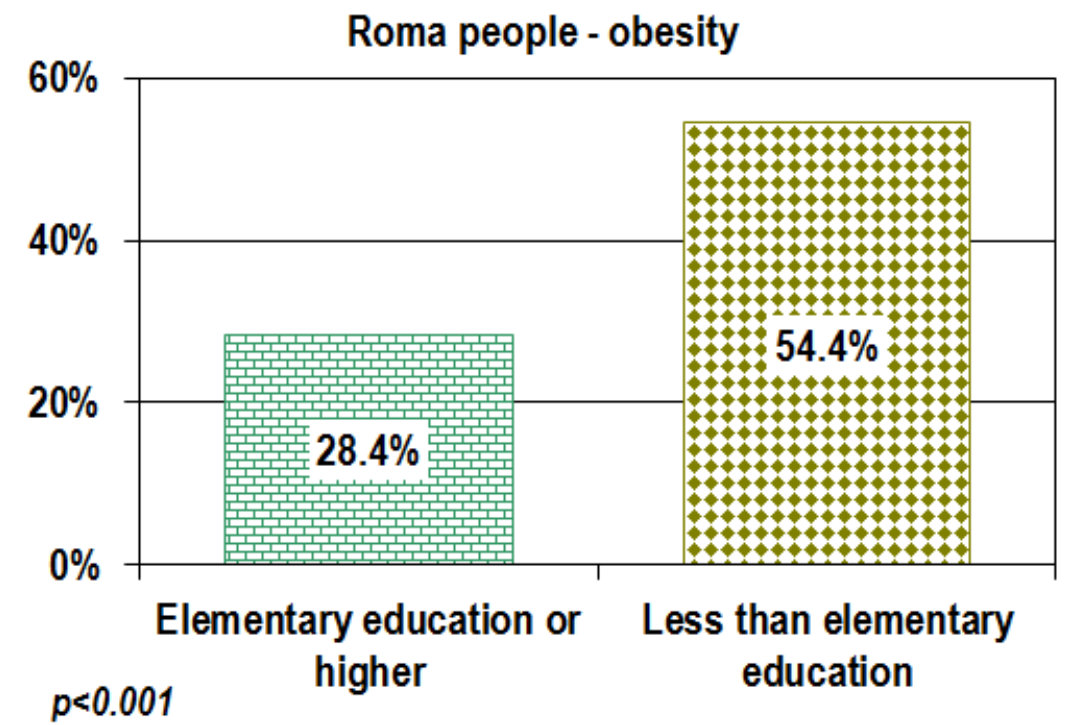

Conclusion: Our results demonstrate high prevalence of diabetes, obesity and hypertension among Roma people in Bulgaria, which are associated with lower of educational level, unemployment and lack of access to medical care.

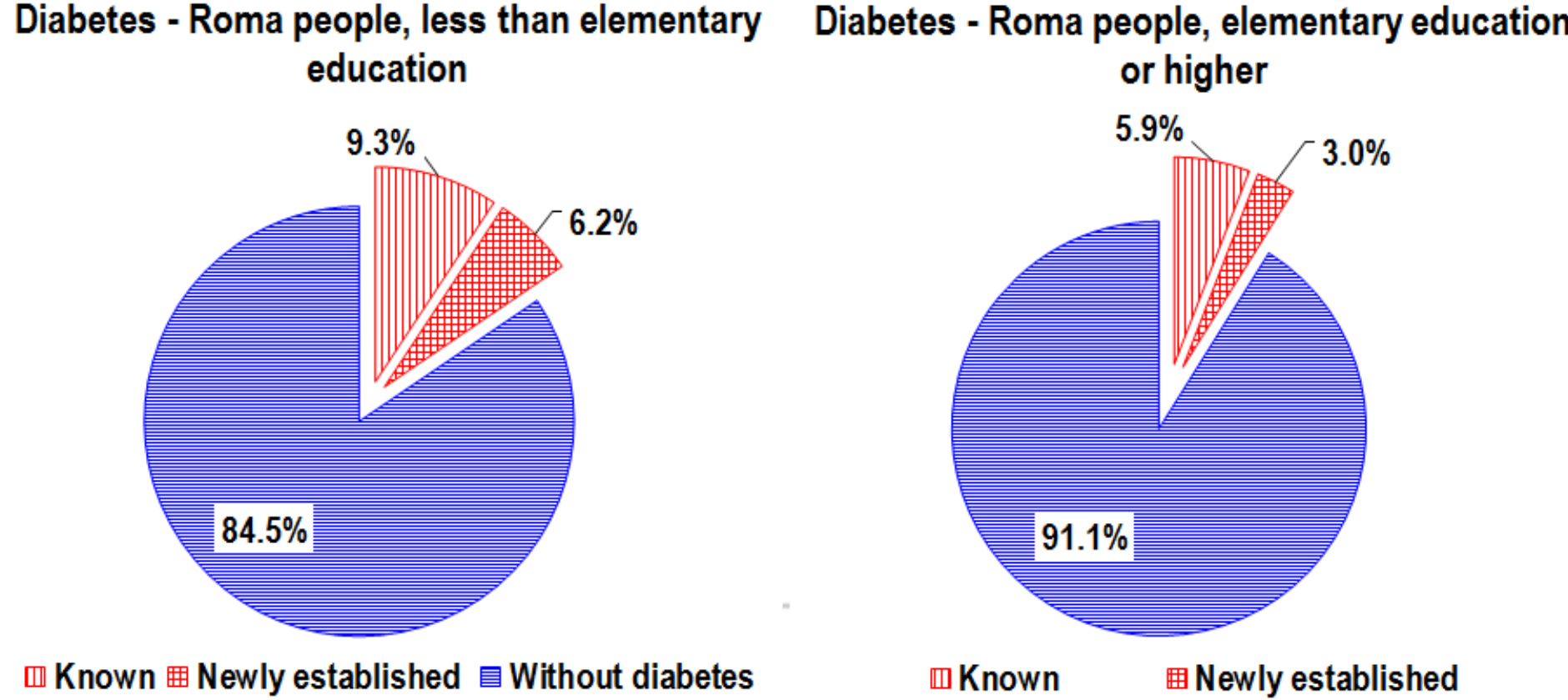

Results: According to BMI, Roma people were distributed as follows: normal BMI - 30.7\%; overweight $-33.2 \%$ and obese $-36.2 \%$. Diabetes was found in $11.2 \%$ of the Roma people $(7.1 \%$ known and $4.1 \%$ newly diagnosed). Arterial hypertension was found in $43.5 \%$ of them. Roma people were divided into two groups considering their educational level and social status. Individuals with less than elementary school fell in group 1. All of them were unemployed. In group 2 are those with elementary or upper education.

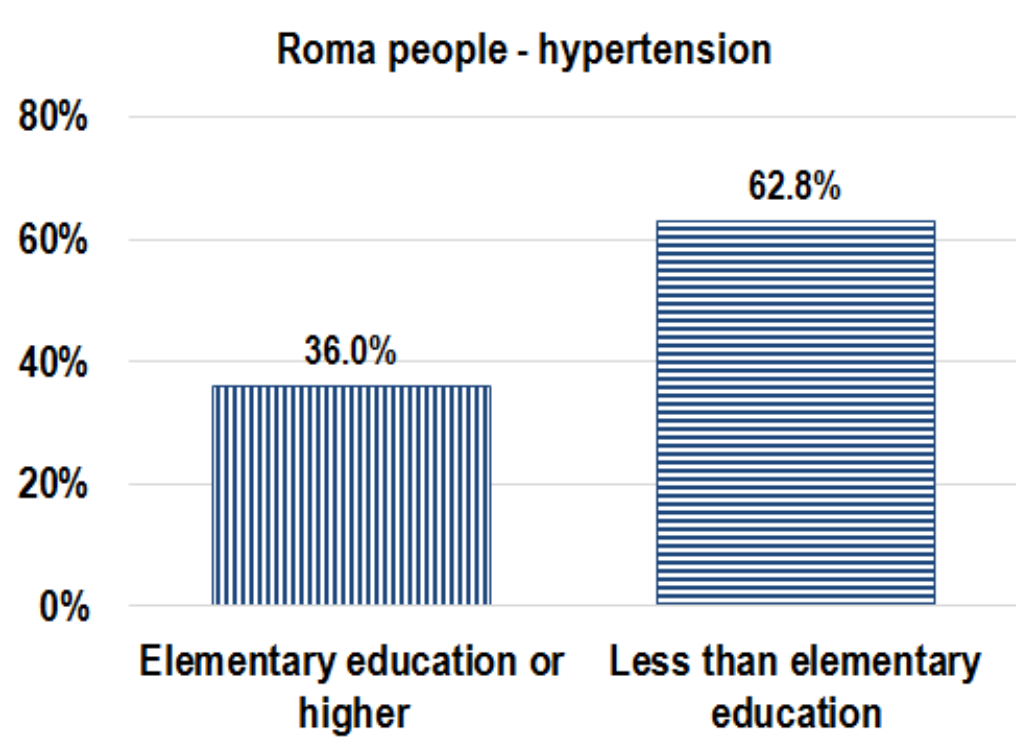

